\title{
Contração de Lorentz, Lei de Gauss e Lei de Ampère
}

\author{
Lorentz Contraction, Gauss and Ampère Laws \\ Olivia Ortiz* \\ Grupo de Óptica/Departamento de Física, Universidade do Estado de Santa Catarina, 89223-100, Joinville, SC, Brazil \\ F. D. Sasse $\mathrm{S}^{\dagger}$ \\ Departamento de Matemática, Universidade do Estado de Santa Catarina, 89223-100, Joinville, SC, Brasil
}

Recebido em 24 de abril, 2003. Aceito em 09 de junho, 2003.

\begin{abstract}
Mostramos como obter a expressão para a contração de Lorentz dos comprimentos, a partir das leis de Gauss e Ampère, sem qualquer conhecimento da teoria da relatividade especial de Einstein. Mostramos em seguida que tal resultado é consistente com a transformação relativística dos campos elétrico e magnético.
\end{abstract}

We show how to obtain the expression for the Lorentz length contraction, using only Gauss and Ampère laws, assuming no previous knowledge of Einstein's theory of special relativity. We also show that this result is consistent with the relativistic transformation of the electric and magnetic fields.

\section{Introdução}

Este artigo tem o objetivo de descrever a professores e alunos de disciplinas básicas de física universitária, como a contração dos comprimentos de Lorentz pode ser entendida como uma solução para um aparente paradoxo originado pela incompatibilidade entre o eletromagnetismo e a mecânica clássica.

A Seção II brevemente descreve como aparentes assimetrias da teoria eletromagnética puderam se constituir numa das principais motivações para a construção da Teoria da Relatividade Especial (TRE).

Na Seção III é mostrado como a hipótese da contração de Lorentz é capaz de resolver uma aparente assimetria decorrente da descrição do movimento de uma partícula-teste carregada nas proximidades de um fio longo, em diferentes referenciais inerciais. Embora tal assunto já tenha sido tratado em textos didáticos de diferentes formas $[1,2,3]$, acreditamos que a presente abordagem tem a virtude de ser bastante simples e acessível, não assumindo a validade da teoria da relatividade especial a priori.

A Seção IV, que supõe que o leitor conhece os fundamentos básicos da TRE, mostra como a formulação relativística do problema fornece a mesma solução, através de uma transformação de Lorentz do tensor eletromagnético, sem necessidade de consideração das fontes.

\section{Relatividade Especial e Eletromag- netismo}

No seu artigo de 1905 sobre a TRE [4], Einstein começa chamando atenção para a assimetria que ocorre na descrição do movimento relativo entre magnetos e condutores. $\mathrm{O}$ artigo não menciona explicitamente qualquer um dos experimentos conflitantes com as teorias vigentes, inclusive o de Michelson-Morley. A primeira frase do artigo afirma: "é sabido que a eletrodinâmica de Maxwell - na forma entendida atualmente - quando aplicada a corpos em movimento, - conduz a assimetrias que não parecem ser inerentes aos fenômenos".

Einstein considerou o problema de uma corrente elétrica induzida em um condutor por um magneto. Tal corrente pode ser induzida de duas formas:

- O magneto é mantido em repouso com relação ao laboratório e um condutor move-se perto dele.

- O magneto move-se e o condutor é mantido em repouso.

No primeiro caso, segundo a formulação clássica da eletrodinâmica de Maxwell, a corrente é induzida porque o movimento das cargas sob o campo magnético do magneto origina uma força sobre cada elétron livre (de carga $e$ ) do condutor, perpendicular ao campo magnético $\mathbf{B}$ e velocidade $\mathbf{v}$ do elétron.

\footnotetext{
*oliviaortiz@ig.com.br

${ }^{\dagger}$ fsasse@joinville.udesc.br
} 
No segundo caso, classicamente a corrente é considerada como sendo produzida por um campo magnético variável, de acordo com a lei de indução de Faraday.

Embora o resultado seja o mesmo, as equações básicas utilizadas para resolver o problema nos dois casos são diferentes.

Tal assimetria era inaceitável para Einstein. Em parte, para resolver tal problema, em vez de tentar alterar diretamente a teoria eletromagnética ou a teoria de Newton, ou supor propriedades atômicas especiais de corpos movendose num éter, ele concluiu que uma grande mudança era necessária na física.

Einstein baseou sua TRE, em dois postulados, enunciados na seguinte forma:

\section{Todas as leis da física têm a mesma forma em qualquer referencial inercial.}

\section{A velocidade da luz no vácuo independe da velocidade da fonte.}

Com seus dois postulados, mais a hipótese de isotropia do espaço, Einstein pode construir a nova mecânica relativística, deixando intacta a teoria de Maxwell. No entanto, a forma do segundo postulado revela a preocupao de Einstein em não assumir a hipótese da estrita validade das equações de Maxwell. Além disso, a contração dos comprimentos e a dilatação dos tempos, que antes eram hipóteses das teorias de éter de Fitzgerald [5], Larmor [6] e Lorentz [7], passaram a ser meras conseqüências desses postulados, não havendo a necessidade de especulações sobre a natureza das forças de coesão dentro de corpos, como réguas e relógios. Também as transformações de Lorentz, apresentadas em 1904 por Poincaré $[8,9]$, puderam ser recuperadas. As aparentes assimetrias, envolvendo eletromagnetismo e trocas de referenciais, desapareceram sob a luz da nova mecânica.

Convém notar ainda que, apesar do triunfo de Einstein, Pauli [10], Bell [11] e Brown [12], observaram que não é claro que o ponto de vista atomístico de Lorentz deva ser abandonado. Uma analogia frequentemente utilizada é a da segunda lei da termodinâmica aplicada à teoria de gases. Embora esta lei possa ser utilizada para explicar e prever vários fenômenos, ela não exclui a necessidade da utilização da teoria cinética (molecular) dos gases [13].

\section{Contração de Lorentz via Lei de Gauss e de Ampère}

Veremos agora como a hipótese da contração dos comprimentos de Lorentz pode ser utilizada para a resolução de um aparente paradoxo em um problema eletromagnético simples. Não vamos assumir, neste ponto, qualquer informação vinda diretamente da teoria da relatividade especial.

Consideremos a seguinte situação física, descrita em um referencial inercial $S$ : uma partícula-teste com carga $q>0$ está situada a uma distância $r$ (medida ao longo do eixo cartesiano $y$ ) de um fio longo e reto, eletricamente neutro, que conduz uma corrente elétrica $I$, na direção positiva do eixo $x$. Neste referencial a carga-teste está em repouso.

Vamos supor ainda que tal corrente é causada pelo deslocamento efetivo de portadores de carga negativa, com velocidade $-v \mathbf{i}$ (i $, \mathbf{j}, \mathbf{k}$ são vetores unitários dirigidos ao longo dos eixos $x, y$ e $z$, respectivamente).

Como o fio é neutro e a carga-teste está em repouso, nenhuma força sobre a partícula é medida no referencial $S$.

Consideremos agora a mesma situação física, agora descrita em um referencial $S^{\prime}$, que se move com velocidade $v \mathbf{i}$, com relação $S$. Em tal referencial a partícula-teste agora move-se com velocidade - vi , assim como os portadores de carga positiva, que agora podem ser considerados como sendo os causadores da corrente $I$. A lei de Ampère agora implica que o campo magnético sobre a partícula-teste é dada por $\mathbf{B}^{\prime}=-B^{\prime} \mathbf{k}$, onde ${ }^{1}$

$$
B^{\prime}=\frac{2 I^{\prime}}{c r},
$$

onde $I^{\prime}=\lambda_{+}{ }^{\prime} v$ é a corrente elétrica agora causada pelo deslocamento da linha de cargas positiva. Como a partícula está em movimento, deve agora atuar sobre ela uma força magnética atrativa, dada pela parte magnética da força de Lorentz, $\mathbf{F}_{\mathbf{m}}=-F_{m} \mathbf{j}$, onde

$$
\mathbf{F}_{\mathbf{m}}=-\frac{q v B^{\prime}}{c} \mathbf{j}=-\frac{q v 2 I^{\prime}}{c^{2} r} \mathbf{j}=-\frac{2 q \lambda_{+}{ }^{\prime} v^{2}}{c^{2} r} \mathbf{j} .
$$

Por outro lado, a simetria do problema indica que, com relação ao eixo $y$, a partícula-teste continua em repouso. A questão natural que surge é: qual a origem da força que contrabalança $\mathbf{F}_{\mathbf{m}}$ ?

Uma hipótese razoável é que no referencial $S^{\prime}$ aparece também uma força elétrica repulsiva $\mathbf{F}_{\mathbf{e}}=F_{e} \mathbf{j}$, causada pelo surgimento de uma densidade de carga líquida $\Delta \lambda^{\prime}$ no fio. Com tal suposição o campo elétrico na posição da cargateste é dado, de acordo com a lei de Gauss, por $\mathbf{E}^{\prime}=E^{\prime} \mathbf{j}$, onde

$$
E^{\prime}=\frac{2 \Delta \lambda^{\prime}}{r}
$$

A força elétrica é então dada por

$$
\mathbf{F}_{\mathbf{e}}=q \mathbf{E}^{\prime}=q E^{\prime} \mathbf{j}=\frac{2 q \Delta \lambda^{\prime}}{r} \mathbf{j} .
$$

Imediatamente podemos observar que $\Delta \lambda^{\prime}>0$.

Como a carga-teste está em equilíbrio, devemos ter

$$
\mathbf{F}_{\mathbf{m}}+\mathbf{F}_{\mathbf{e}}=0
$$

ou seja,

$$
-\frac{2 q \lambda_{+} v^{2}}{c^{2} r}+\frac{2 q \Delta \lambda^{\prime}}{r}=0 .
$$

\footnotetext{
${ }^{1}$ Em todo este artigo utilizamos o sistema gaussiano de unidades
} 
Seja $\lambda_{ \pm}{ }^{\prime}$ a densidade de cargas positivas (negativas) no fio, medida em $S^{\prime}$. Então

$$
\Delta \lambda^{\prime}=\lambda_{+}{ }^{\prime}+\lambda_{-}{ }^{\prime} .
$$

A eq. (6) pode agora ser escrita como

$$
-\frac{v^{2}}{c^{2}} \lambda_{+}{ }^{\prime}+\lambda_{+}{ }^{\prime}+\lambda_{-}{ }^{\prime}=0 .
$$

ou

$$
\lambda_{-}{ }^{\prime}=-\lambda_{+}{ }^{\prime} / \gamma^{2},
$$

onde

$$
\gamma:=\frac{1}{\sqrt{1-v^{2} / c^{2}}} .
$$

Como estamos supondo que houve uma mudança de densidade de cargas na passagem de um referencial inercial a outro, a simetria do problema sugere que

$$
\lambda_{+}{ }^{\prime}=k \lambda_{+},
$$

onde $k$ é uma constante de proporcionalidade e $\lambda_{+}$é a densidade de cargas positivas no referencial inercial $S$. Por simetria devemos ter também

$$
\lambda_{-}{ }^{\prime}=\frac{\lambda_{-}}{k} .
$$

Por hipótese, o fio é eletricamente neutro em $S$, de modo que $\lambda_{+}=-\lambda_{-}$. Dividindo (12) por (11) e comparando com (9), obtemos

$$
k=\gamma=\frac{1}{\sqrt{1-v^{2} / c^{2}}} .
$$

Uma vez que a carga total deve ser conservada, vemos que a carga total em um segmento do fio deve ser a mesma nos dois referenciais, ou seja,

$$
Q_{ \pm}=\lambda_{ \pm} L_{ \pm}=\lambda_{ \pm}{ }^{\prime} L_{ \pm}^{\prime}
$$

onde $Q_{ \pm}$é a carga total positiva (negativa) em um segmento do fio com comprimento $L_{ \pm}$, em $S$, e $L_{ \pm}{ }^{\prime}$, em $S^{\prime}$.

Portanto, de acordo com as eqs. (11), (12) e (13), a equação acima implica que

$$
L_{+}{ }^{\prime}=\frac{L_{+}}{\gamma}, \quad L_{-}{ }^{\prime}=\gamma L_{-} .
$$

Como $\gamma>1$, vemos que a linha de cargas positivas, que passou do repouso ao movimento, sofreu uma contração no seu comprimento, na passagem do referencial $S$ para $S^{\prime}$. Por outro lado, a linha de cargas negativas, que passou do movimento ao repouso, sofreu uma dilatação.

Embora tais conclusões sejam consequências simples das transformações de Lorentz, nossa ênfase aqui consiste no fato de que elas podem ser obtidas de forma muito simples, invocando somente a validade das equações de Maxwell, em particular leis de Gauss e Ampère, em qualquer referencial inercial.

\section{Formalismo Covariante}

Quando apresentada na sua forma covariante a eletrodinâmica de Maxwell explica naturalmente o fenômeno descrito anteriormente, em dois referenciais inerciais, sem a necessidade da consideração das fontes.

No que se segue utilizamos índices latinos para denotar componentes espaciais de tensores, $i, j, k, \ldots=1,2,3$, e índices gregos para denotar componentes espaço-temporais $\mu, \nu, \alpha, \ldots=0,1,2,3$. Índices de componentes repetidos superiores e inferiores subentendem um somatório. Exceto pelas componentes dos campos elétrico e magnético, grandezas denotadas, por letras maiúsculas referem-se a 4vetores e 4-tensores, de acordo com a convenção de Rindler [14].

Vamos supor que associado ao referencial inercial $S$ temos um sistema de coordenadas $x^{\mu}=(c t, x, y, z)$ e ao referencial $S^{\prime}$ o sistema $x^{\mu \prime}=\left(c t^{\prime}, x^{\prime}, y^{\prime}, z^{\prime}\right)$.

A força de Lorentz sobre uma partícula carregada tem a seguinte forma covariante

$$
\frac{d P^{\mu}}{d \tau}=\frac{q}{c} F^{\mu \nu} U_{\nu},
$$

$\tau$ é o tempo próprio da partícula, $P^{\mu}=m U^{\mu}$ é o 4-momento, $U^{\mu}=d X^{\mu} / d \tau$ a 4-velocidade, $X^{\mu}=$ $(c t, x, y, z)$ o 4 -vetor posição, $t$ o tempo do laboratório, com $d t=\gamma(v) d \tau . \quad m$ a massa de repouso da partícula, $\gamma(v)=1 / \sqrt{1-v^{2} / c^{2}}, \mathbf{v}$ a 3-velocidade, $U_{\nu}:=\eta_{\mu \nu} U^{\mu}$, $\eta_{\mu \nu}=\operatorname{diag}(1,-1,-1,-1)$, e $F^{\mu \nu}$ é o 4-tensor de segunda ordem antissimétrico, denominado tensor eletromagnético, definido por [15]

$$
\left[F^{\mu \nu}\right]=\left(\begin{array}{cccc}
0 & -E_{x} & -E_{y} & -E_{z} \\
E_{x} & 0 & -B_{z} & B_{y} \\
E_{y} & B_{z} & 0 & -B_{x} \\
E_{z} & -B_{y} & B_{x} & 0
\end{array}\right)
$$

As componentes espaciais da força de Lorentz são dadas por

$$
\frac{d P^{i}}{d \tau}=\frac{q}{c} F^{i \mu} U_{\mu}=\frac{q}{c} F^{i 0} U_{0}+\frac{q}{c} F^{i j} U_{j} .
$$

As componentes covariantes $U_{\mu}=\left(U_{0}, U_{j}\right)$ da 4velocidade são

$$
\begin{aligned}
U_{0} & =\eta_{00} \frac{d X^{0}}{d \tau}=\gamma c \\
U_{i} & =\eta_{i \mu} \frac{d X^{\mu}}{d \tau}=\eta_{i i} \frac{d x^{i}}{d t} \gamma=-\gamma v^{i} .
\end{aligned}
$$

No referencial $S$, temos, $v^{i}=0, E^{i}=0$ de modo que de (18) temos $d P^{i} / d \tau=0$.

No referencial $S^{\prime}, v^{i^{\prime}}=(-v, 0,0)$ e as componentes dos campos elétrico e magnético podem ser obtidas examinando as componentes do tensor eletromagnético $F^{\mu \nu^{\prime}}$, obtidas a transformação de Lorentz

$$
F^{\mu \nu^{\prime}}=\Lambda_{\alpha}^{\mu^{\prime}} \Lambda_{\beta}^{\nu^{\prime}} F^{\alpha \beta},
$$


onde

$$
\left[\Lambda_{\alpha}^{\mu^{\prime}}\right]=\left(\begin{array}{cccc}
\gamma & -\gamma v / c & 0 & 0 \\
-\gamma v / c & \gamma & 0 & 0 \\
0 & 0 & 1 & 0 \\
0 & 0 & 0 & 1
\end{array}\right)
$$

é a matriz de boost de Lorentz na direção $x$.

A única componente não nula de $F^{\mu \nu}$ é $F^{21}=-F^{12}=$ $B_{z}$. Portanto, as componentes não nulas de $F^{\mu \nu^{\prime}}$ são $F^{02^{\prime}}=$ $E_{y}{ }^{\prime}$ e $F^{12^{\prime}}=-B_{z}{ }^{\prime}$, onde

$$
\begin{aligned}
F^{02^{\prime}} & =\Lambda^{0^{\prime}}{ }_{\alpha} \Lambda^{2^{\prime}}{ }_{\beta} F^{\alpha \beta}=\Lambda^{0^{\prime}}{ }_{1} \Lambda^{2^{\prime}}{ }_{2} F^{12} \\
& =-\frac{\gamma v B_{z}}{c}=-\frac{2 \gamma I^{\prime} v}{c^{2} r}
\end{aligned}
$$

$\mathrm{e}$

$$
\begin{aligned}
F^{12^{\prime}} & =\Lambda^{1^{\prime}}{ }_{\alpha} \Lambda^{2^{\prime}}{ }_{\beta} F^{\alpha \beta}=\Lambda^{1^{\prime}}{ }_{1} \Lambda^{2^{\prime}}{ }_{2} F^{12} \\
& =-\gamma B_{z}=-\frac{2 \gamma I^{\prime} v}{c r}
\end{aligned}
$$

Substituindo (19), (20), (23) e (25) na expressão para a força de Lorentz (18), obtemos imediatamente

$$
\frac{d P^{i^{\prime}}}{d \tau}=0,
$$

ou seja, a força resultante sobre a partícula carregada permanece nula em $S^{\prime}$, como esperado.

\section{Conclusões}

Mostramos nesse trabalho como deduzir facilmente a expressão para a contração de Lorentz dos comprimentos, utilizando somente as leis de Gauss e Ampère.

Tal exemplo mostra que efeitos relativísticos podem ser importantes mesmo quando baixas velocidades são envolvidas. As velocidades efetivas de elétrons em um fio condutor são tipicamente da ordem de $10^{-5} \mathrm{~m} / \mathrm{s}$, para um fio de cobre calibre 14 . Isso implica que $v / c$ é extremamente pequeno, e $\gamma(v)$ muito próximo a 1 , resultando numa contração de comprimentos muito pequena. No entanto, os efeitos relativísticos são importantes, pois as densidades de cargas envolvidas são muito altas, tipicamente $10^{22} / \mathrm{m}^{3}$ para o cobre.

Possíveis extensões do método descrito na Seção III podem ser aplicadas a outros sistemas. Um caso interessante envolve o cálculo do campo magnético de um anel condutor. Uma corrente induzida em um anel inicialmente neutro daria origem não somente a um campo magnético, mas também a um campo elétrico decorrente do aparecimento de uma densidade de carga elétrica líquida, decorrente da contração da linha de cargas negativas. O método covariante, apresentado na Seção IV, aplicado ao mesmo problema conduz a um aparente paradoxo.

A análise covariante também conduz a resultados interessantes quando utilizada para descrever o campo eletromagnético em referenciais não-inerciais, de acordo com o princípio da localidade $[16,17]$. Tais problemas serão abordados em um futuro trabalho.

\section{Agradecimentos}

Olivia Ortiz agradece à UDESC pelo apoio financeiro, durante a realização deste trabalho, através da Bolsa de Iniciação Científica PROBIC.

\section{Referências}

[1] Edward M. Purcell, Curso de Física de Berkeley, vol. 2, Eletricidade e Magnetismo, Edgard Blücher Ltda., São Paulo, 1970.

[2] George R. R. Ellis e Ruth M. Williams, Flat and Curved Space-Times, Oxford University Press, Oxford, 1987.

[3] Paul A. Tipler, Física, vol. II, Guanabara Dois, Rio de Janeiro, 1978

[4] Albert Einstein, Zur Elektrodynamik bewegter Körper, Annalen der Physik, 17, 891 (1905).

[5] G. F. FitzGerald, The Ether and the Earthś Atmosphere, Science, 13, 390 (1889).

[6] Joseph Larmor, Aether and Matter, Cambridge University Press, Cambridge, UK, 1900.

[7] H. A. Lorentz, Electromagnetic Phenomena in a System Moving with Any Velocity Smaller Than That of Light. In Proceedings of the Section of Sciences, Kon. Akademie van Wettenshappen, volume 6, pages 809-831, Amsterdam, 1904, English-language edition.

[8] J. H. Poincaré, Létat actuel et lávenir de la physique mathématique, Bulletin des Sciences Mathéematiques, première partie, 17, 302-324, (1904). The English translation is in Poincaré, 1905.

[9] J. H. Poincaré, “ The Principles of Mathematical Physics; Monist, 15(1), 1-24 (1905).

[10] W. Pauli, Theory of Relativity, Dover Pub. Inc., New York, N.Y., 1981.

[11] J. S. Bell, Speakable and unspeakable in quantum mechanics, Cambridge University Press, Cambridge, UK, 1987.

[12] Harvey R. Brown and Oliver Pooley, "The origin of the spacetime metric: Bellś Lorentzian pedagogy. In Physics meets Philosophy at the Planck Scale, Cambridge, UK, 2000. Cambridge University Press.

[13] Gerald Holton, Thematic Origins of Scientific Thought - Kepler to Einstein, Harvard University Press, Cambridge, Massachusets and London, England, 1988.

[14] Wolfgand Rindler, Relativity - Special, General and Cosmological, Oxford University Press, Oxford, 2001.

[15] J. D. Jackson, Classical Electrodynamics, John Wiley and Sons, 3rd Ed., New York, 1998.

[16] W. M. Irvine, Electrodynamics in a Rotating System of Reference, Physica, 30, 1160 (1964).

[17] Bahram Mashhoon, On the Relativity of Rotation In B. L. Hu and T. A. Jacobson, editors, Directions in General Relativity, volume 2, pages 182-193, Cambridge University Press, 1993. Papers in honor of Dieter Brill. 